\title{
THE RECOGNITION AND INCIDENCE OF HAPLOID AND POLYPLOID SPERMATOZOA IN MAN, RABBIT AND MOUSE
}

\author{
A. D. CAROTHERS* AND R. A. BEATTY $\dagger$
}

Department of Genetics, University of Edinburgh

(Received 20th November 1974)

Summary. The existence of polyploid mammalian spermatozoa has been inferred from studies of Feulgen-DNA absorption. Rabbit spermatozoa fell into two discrete groups with mean absorptions close to a $1: 2$ ratio (inferred to be haploids and diploids respectively); simple visual appraisal of the size of the head or nucleus gave an identical classification. The incidences of ploidy classes were $98.94 \%$ haploid, $1.06 \%$ diploid, $0.00 \%$ higher than diploid $(\mathrm{N}=3010$; from DNA measurements and visual appraisal of the size in a rabbit chosen to have a high incidence of diploids) and, correspondingly, $99.691 \%, 0.308 \%, 0.001 \%$ $(\mathrm{N}=138001$; from sixty-nine unselected rabbits, scored by visual appraisal of the size of the sperm head). In man also, virtually discrete groups with absorptions close to a $1: 2$ ratio existed and were inferred to be haploids and diploids respectively. A few human spermatozoa were found with absorptions corresponding to a ploidy of three and/or four. Visual appraisal of the size of the human sperm nucleus as Small, Medium or Large was only a partial guide to ploidy. All Small human spermatozoa measured for DNA absorption were found to be haploid. About two-thirds of Medium human spermatozoa were found, however, to be haploid, and some Large spermatozoa were haploid or diploid. The incidences of ploidy classes in the human were $99.37 \%$ haploid, $0.56 \%$ diploid, $0.07 \%$ higher than diploid $(\mathrm{N}=5554$; with consistency between duplicate slides and between two subjects; from DNA measurements and visual appraisal of nuclear size). The estimated incidence of diploid human spermatozoa is consistent with the known incidence of triploid fetuses. In a mouse with a putatively high incidence of diploids, all 1000 DNA measurements were nevertheless within the haploid range, with one diploid encountered outside the main sampling.

\section{INTRODUGTION}

Chromosomal abnormality is a cause of prenatal wastage in mammals, particularly in human conceptuses, of which $1.5 \%$ are estimated to have a triploid or (more rarely) a tetraploid constitution (Jacobs, 1972), and about

* Present address: M.R.C. Clinical and Population Cytogenetics Unit, Western General Hospital, Edinburgh.

$\dagger$ Agricultural Research Council Unit of Animal Genetics. 
$1 / 5$ of all spontaneous human abortion due to chromosome abnormality is attributable to triploidy (Carr, 1971; Boué \& Boué, 1974). Study of the origin of triploidy requires an apportionment of responsibility between (a) fertilization of a chromosomally-doubled egg by a normal spermatozoon, (b) fertilization of a normal egg by two spermatozoa, and (c) fertilization of a normal egg by a diploid spermatozoon. In the human, a preliminary calculation by Beatty (1974) suggested that about $40 \%$ of human triploidy was caused by diploid spermatozoa. The present work was undertaken with the feeling that methods for determining the ploidy of spermatozoa required confirmation and extension.

Our main objective was to infer ploidy from frequency distributions of the Feulgen-DNA absorption of individual spermatozoa of rabbit, man and mouse. Spermatozoa can be classified subjectively into Small, Medium or Large according to visual appraisal of the size of the sperm head or the nucleus. Further objectives were to assess how well these size classes corresponded to DNA-scored ploidy, and to estimate the hydrolysis time giving maximum Feulgen-DNA absorption in human spermatozoa.

Strictly speaking, the 'ploidy' terms should refer to the number of chromosomes in a cell nucleus. As with other methods of estimating the bulk DNA content of cell nuclei, however, measurement of Feulgen-DNA absorption cannot determine this number exactly but establishes ranges of values of DNA content corresponding to classes such as near-haploids, or near-diploids. For brevity, we shall refer to these as 'haploid' or ' $1 \mathrm{~N}$ ', and 'diploid' or ' $2 \mathrm{~N}$ ', and the $2 \mathrm{~N}, 3 \mathrm{~N}$ and $4 \mathrm{~N}$ classes will be referred to collectively as 'polyploids', the $3 \mathrm{~N}$ and $4 \mathrm{~N}$ classes as ' $>2 \mathrm{~N}$ '.

\section{MATERIALS AND METHODS}

\section{Spermatozoa}

Ejaculated rabbit semen was collected with an artificial vagina. In order to increase the expected proportion of diploid spermatozoa, a young (35-week-old) male of the AD strain was used (Beatty \& Fechheimer, 1972). Human semen obtained by masturbation was provided by presumptively normal volunteer donors. A mouse semen suspension was obtained by squeezing the contents of the excised ductus deferentes into a drop of $0.9 \% \mathrm{NaCl}$ : again, the expectation of diploid spermatozoa was raised by choosing a young (9-week-old) male of a strain (V-strain, = 'silver strain') that has an association with polyploidy (Beatty, 1957).

\section{Preparations}

Nigrosin-eosin slides of rabbit semen were prepared by the method of Beatty \& Sharma (1960), but one drop of undiluted semen was added to one drop of stain, and they were left in contact for 5 min.

Feulgen staining of rabbit semen was conducted by air-drying the semen smear, fixing for $24 \mathrm{hr}$ in methanol, air-drying, rinsing in $1 \mathrm{~N}-\mathrm{HCl}$ at room temperature for $1 \mathrm{~min}$, hydrolysing in $1 \mathrm{~N}-\mathrm{HCl}$ at $60^{\circ} \mathrm{C}$ for $6 \mathrm{~min}$, rinsing in $1 \mathrm{~N}-\mathrm{HCI}$ for $1 \mathrm{~min}$, passing through three 2-min baths of distilled water, and staining for $2 \mathrm{hr}$ in Feulgen reagent. The preparations were then blotted, 
passed through three 5 -min baths of sulphurous acid solution $\left(1 \mathrm{~g} \mathrm{~K}_{2} \mathrm{~S}_{2} \mathrm{O}_{5}\right.$, $10 \mathrm{ml}$ of $1 \mathrm{~N}-\mathrm{HCl}, 200 \mathrm{ml}$ distilled water), washed in water, dried, and mounted in neutral XAM (Gurr, Improved White, RI 1.5208).

Feulgen staining in Series 1 of human material was conducted as for the rabbit. Feulgen preparations for Series 2 of human material were made by a procedure based on those of Böhm, Sprenger, Schlüter \& Sandritter (1968) and Moore \& Beatty (1974). Thirty air-dried smears, prepared from a single ejaculate, were fixed for $90 \mathrm{~min}$ in a methanol:formalin:acetic acid mixture ( $85: 10: 5$ by vol.), rinsed four times in distilled water, passed through three 5 -min baths of distilled water, dried, and hydrolysed in $5 \mathrm{~N}-\mathrm{HCl}$ at $25^{\circ} \mathrm{C}$. Sets of three replicate slides were hydrolysed for the following times, arranged to be in geometric progression: 10.0, 14.0, 19.7, 27.6, 38.7, 54.3, 76.2, 107 and $150 \mathrm{~min}$. One control set of three slides was not hydrolysed. After hydrolysis, slides were washed for $15 \mathrm{~min}$ in running tap water, rinsed in distilled water, placed in fresh Feulgen reagent at room temperature (about $24^{\circ} \mathrm{C}$ ) for $1 \mathrm{hr}$, rinsed in sulphurous acid solution, passed through three 5-min baths of sulphurous acid solution, rinsed twice in distilled water, dried, mounted in XAM and stored in the dark. Feulgen preparations for Series 3 of human material constituted two of the slides hydrolysed for $38.7 \mathrm{~min}$, a time close to an optimum hydrolysis time of 40.7 min estimated from the data of Series 2 .

Feulgen staining for mouse spermatozoa was carried out as for Series 2 of human material, but with hydrolysis for $40 \mathrm{~min}$.

\section{Photometric measurements of Feulgen-DNA}

Photometric measurements of individual sperm nuclei in Feulgen preparations were made with Barr and Stroud Integrating Microdensitometers, type G.N.2., according to the method of Deeley (1955), and expressed in arbitrary units of Feulgen-DNA absorption. Technical variation, including fading of the stain over time, prevented standardization of the units of measurement between slides. All measurements in the present paper are of relative absorptions, each measurement being the difference between a single reading obtained with the nucleus in the centre of the field and one obtained from an adjacent clear area free of nuclei and foreign bodies. When a particular nucleus was measured more than once, this was achieved by re-centring it, refocussing and retaking its reading after each 'blank' measurement, then taking a reading from a different blank area and calculating the difference. In the human and rabbit series, each reading corresponded to a single scanning of the field. In the mouse series, each reading was taken as the average from two consecutive scannings of the same field (performed without intermediate adjustment), thus helping to compensate for the lesser precision of the densitometer used.

\section{Sampling and visual classification of spermatozoa}

Sperm nuclei in Feulgen preparations were observed under the microscope of the microdensitometer and selected for counting and/or DNA measurement as follows: (i) the slide was moved to a new position without being observed through the eyepiece, and with the first field stop at its maximum aperture; (ii) each nucleus within the selected field was examined in turn and classified 
as 'acceptable' if a circle, with centre at the centre of its head and radius equal to that of the working aperture of the first field stop, did not contain other nuclei or parts of nuclei or conspicuous foreign bodies, and did not overlap the edge of the outer field; (iii) each 'acceptable' nucleus within the field was classified as Small, Medium or Large according to its size judged subjectively and was then either measured for DNA or simply counted.

Spermatozoa in nigrosin-eosin preparations were observed under a conventional optical microscope and selected for counting as follows: (i) the slide was moved to a new position without being observed through the eyepiece; (ii) each spermatozoon having the centre of its head within the field was classified as Small, Medium or Large according to the size of its head judged subjectively and was counted.

These criteria of selection, being independent of the size and quality of spermatozoa or of their nuclei, removed a possible source of observer bias. For Feulgen preparations, the method of selection also ensured that all 'acceptable' nuclei were measured without interference from neighbouring bodies. The terms 'Small', 'Medium' and 'Large' do not refer to measurements of Feulgen-DNA absorption.

\section{Rabbit}

\section{RESULTS}

One Feulgen-stained slide and two nigrosin-eosin slides were prepared from a single ejaculate.

Observations were made on 1010 sperm nuclei. The absorption of each Small nucleus was determined ('first measurements') with a duplicate determination on every fifth Small nucleus ('second measurements'). The absorption of each Medium nucleus was determined as the average of four measurements. There were no Large nuclei. The 'first measurements' of the Small nuclei and the average measurements of the Medium nuclei fell into two discrete groups containing 999 and 11 nuclei respectively (Text-fig. 1), giving estimated means of $30.00 \pm 0.05$ (998 d.f.) and 58.12 \pm 0.98 (10 d.f.) respectively. The estimated means were in a ratio that did not differ significantly from an expected haploid: diploid ratio of $1: 2$ (Behren's test; $P=0.10$ to 0.05 ; see Table VI 2 of Fisher \& Yates, 1963). The two groups were regarded as corresponding to $1 \mathrm{~N}$ and $2 \mathrm{~N}$ nuclei, the incidence of the latter being estimated as 1.09 ( \pm about 0.33 ) \%, and no nuclei had an absorption corresponding to $>2 \mathrm{~N}$. Without exception, every Small nucleus had an absorption within the $1 \mathrm{~N}$ range and every Medium nucleus a value within the $2 \mathrm{~N}$ range. Evidently, $1 \mathrm{~N}$ and $2 \mathrm{~N}$ spermatozoa in this sample could be distinguished from one another without error either by relative absorption or by visual appraisal of relative size, and the two methods concurred in showing an absence of $>2 \mathrm{~N}$ spermatozoa. Further evidence for the agreement between the two methods came from eleven nuclei, not included in the random count, which were classed as Medium and measured. Their absorption measurements ranged from 52.9 to 77.9 , with a mean of $61 \cdot 73$, and all were assumed to be $2 \mathrm{~N}$.

Visual classification of 1000 sperm heads on each of the two nigrosin-eosin slides into Small, Medium or Large yielded the numbers 992:8:0 and 987:13:0. 


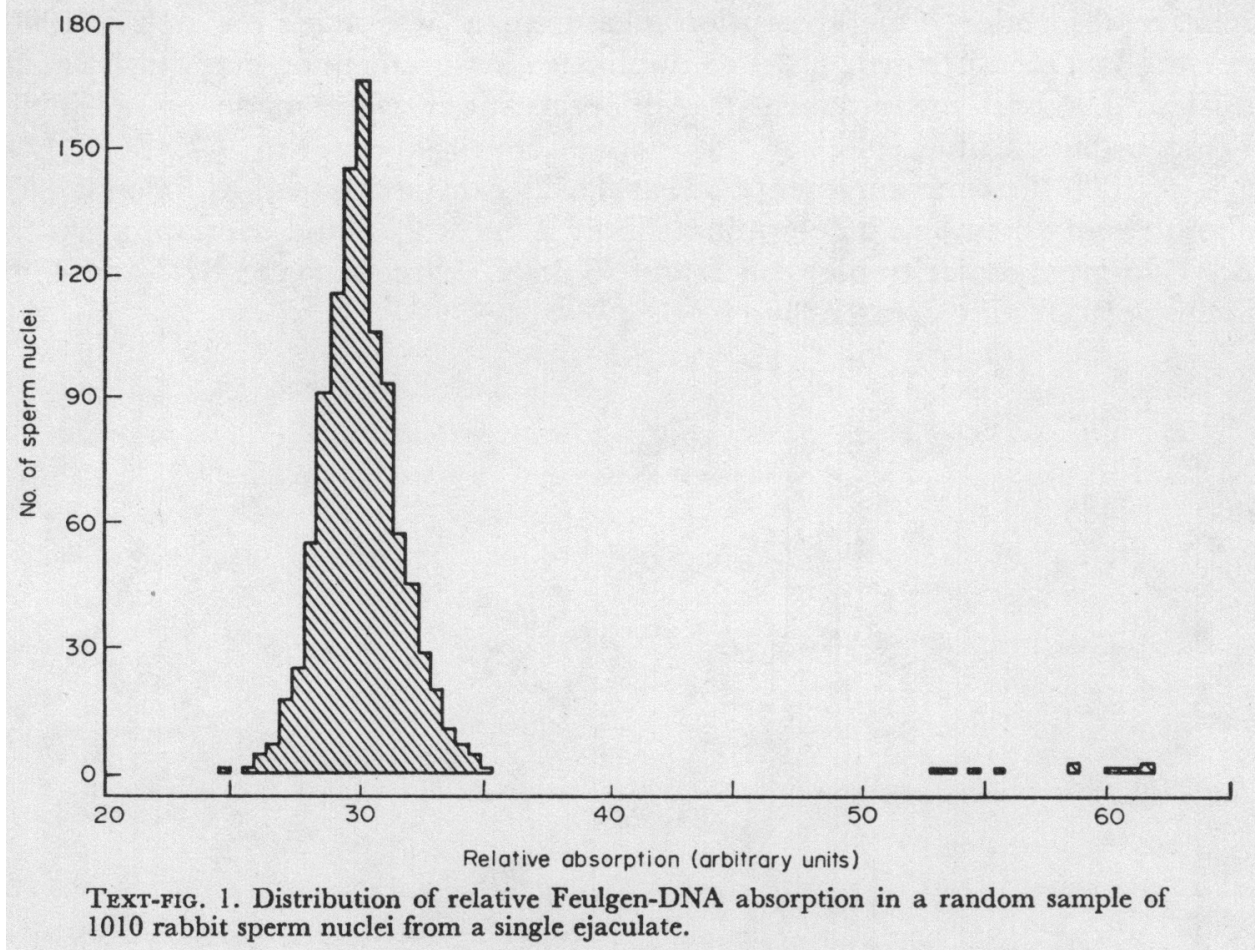

These numbers are consistent with one another and with the proportions of $1 \mathrm{~N}$, $2 \mathrm{~N}$ and $>2 \mathrm{~N}$ nuclei recorded in the previous paragraph, and show that the ploidy of spermatozoa could be scored with the same degree of accuracy by visual appraisal of the size of the nucleus or of the whole sperm head. The consistency between the results of visual appraisal and absorption measurements means that the separate figures may be combined, giving $29781 \mathrm{~N}$ spermatozoa, thirty-two $2 \mathrm{~N}$, and none $>2 \mathrm{~N}(98.94,1.06$ and $0 \%)$.

Sources of variation in the measured absorption of individual nuclei were explored by an analysis of variance. If the 'first measurements' of the $999 \mathrm{lN}$ nuclei are grouped into batches of approximately fifty consecutive nuclei, and if the 'second measurements' are incorporated, then the relative importance of the following sources of variation is illustrated by the percentage variance components given in parentheses: variation including real differences in DNA content of $1 \mathrm{~N}$ nuclei but excluding the two following categories, $57 \%$, differences between batches of size fifty, $28 \%$, lack of repeatability of measurements on the same nuclei, $15 \%$. The $28 \%$ estimate differs from zero with a high degree of significance.

The distribution of $1 \mathrm{~N}$ absorption measurements differed significantly from the Normal as judged by a $\chi^{2}$ goodness-of-fit test $(P<0 \cdot 001,15$ d.f. $)$.

\section{Human spermatozoa, Series 1}

From other observations, the hydrolysis procedure was known not to yield maximum absorption, but was assumed to be adequate for consideration of 
relative absorption. One absorption measurement was made on each of 1000 nuclei ('first measurement'), with a duplicate measurement on every fifth Small nucleus ('second measurement'). All observations were made on a single Feulgen-stained slide.

The 1000 'first measurements' (Text-fig. 2) can be grouped as follows: (A) about twenty below an indeterminate limit, L, near 23.0 and overlapping with B; (B) a great majority between $\mathrm{L}$ and 33.0 ; (C) three between 37.0 and 43.0 inclusive; (D) nine greater than $52 \cdot 0$.

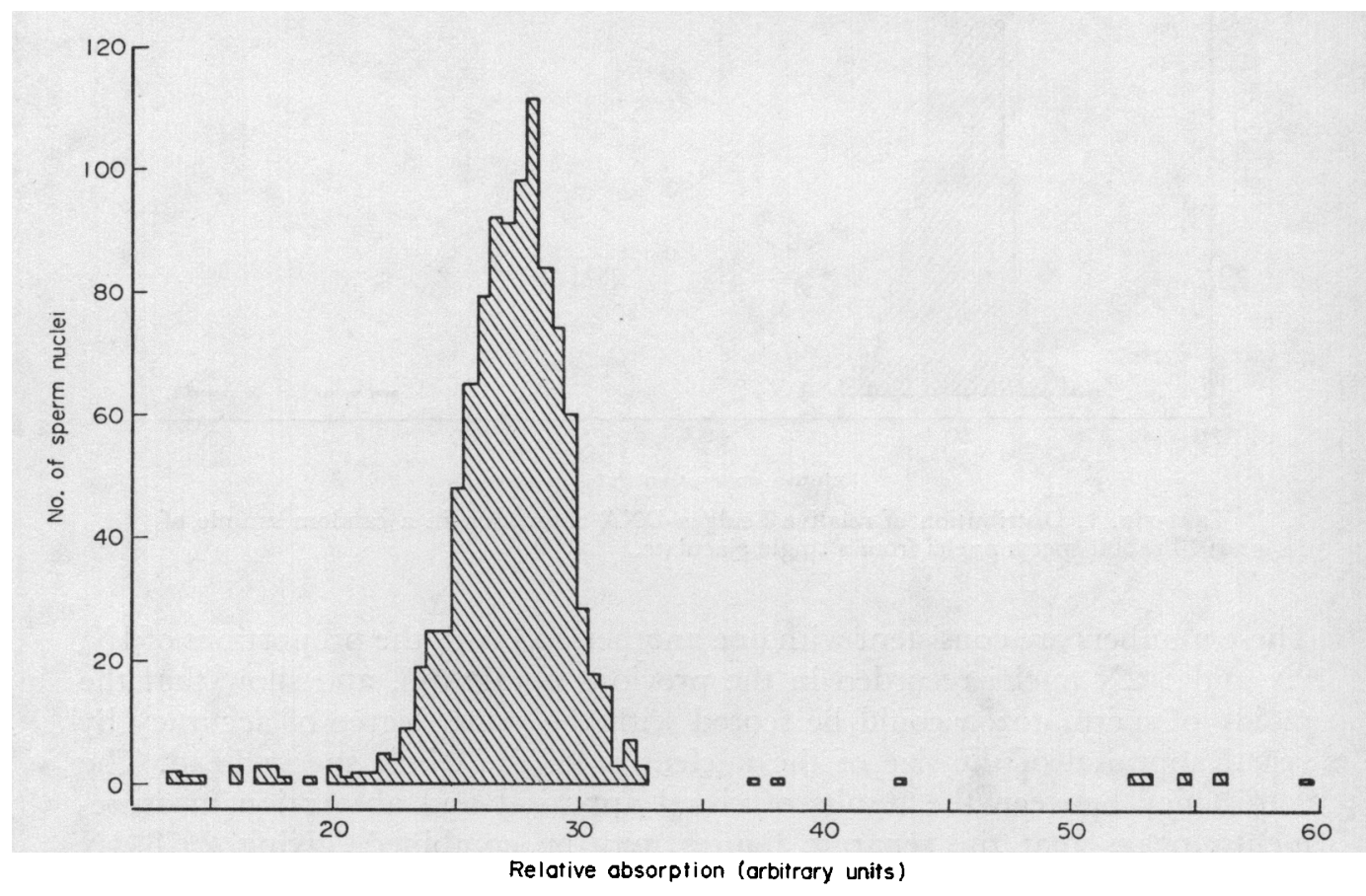

Text-Fig. 2. Series 1. Distribution of relative Feulgen-DNA absorption in a random sample of 1000 human sperm nuclei from a single ejaculate.

To resolve $\mathrm{A}$ and $\mathrm{B}$, the assumption was made that no member of $\mathrm{A}$ lay above $L$ and a truncated Normal curve was then fitted to the set of measurements between $(L+0.5)$ and 33.0 inclusive, i.e. to the $B$ data. Taking $L$ to be 23.0 , an iterative maximum likelihood procedure yielded an estimate of $27.29 \pm 0.06$ for the mean of the B population. A Normal curve constructed from these estimates gave a very good fit for the $\mathrm{B}$ data $\left(\chi^{2}=16\right.$ on 18 d.f.; $P=0.2$ to 0.5$)$. The estimated mean of the $\mathrm{D}$ population was $54.61 \pm 0.76$ ( 8 d.f.), or almost exactly twice that of $B$. The value of $L$ was, of course, arbitrary, but variations of its value within commonsense limits had trivial effects on the estimated mean and standard error of $B$.

A likely interpretation is that $\mathrm{A}$ and $\mathrm{C}$ included 'abnormal' $1 \mathrm{~N}$ nuclei, $\mathrm{B}$ included only $1 \mathrm{~N}$, while $\mathrm{D}$ was constituted by nine $2 \mathrm{~N}$. On this basis, the incidence of $2 \mathrm{~N}$ was estimated as 0.90 ( \pm about 0.30 ) \%. 
All the Small nuclei had absorptions in the $1 \mathrm{~N}$ range, but nineteen of the Medium nuclei were in the $1 \mathrm{~N}$ range and nine in the $2 \mathrm{~N}$ range (Table 1 ). Evidently, all Small nuclei were $1 \mathrm{~N}, 32 \%$ of Medium nuclei were $2 \mathrm{~N}$ (the remaining $68 \%$ being $1 \mathrm{~N}$ ), and an absence of Large nuclei or of those with an absorption corresponding to $>2 \mathrm{~N}$ indicated an absence of $>2 \mathrm{~N}$ spermatozoa in this sample.

Table 1. Summary of results on the ploidy of human spermatozoa

\begin{tabular}{|c|c|c|c|c|c|}
\hline & & \multicolumn{3}{|c|}{ Ploidy } & \multirow[b]{2}{*}{ Total } \\
\hline & & $1 \mathcal{N}$ & $2 \mathcal{N}$ & $>2 \mathcal{N}$ & \\
\hline & $\begin{array}{l}\text { Series } 1 \\
\text { Small } \\
\text { Medium } \\
\text { Large }\end{array}$ & $\begin{array}{r}972 \\
19 \\
0\end{array}$ & $\begin{array}{l}0 \\
9 \\
0\end{array}$ & $\begin{array}{l}0 \\
0 \\
0\end{array}$ & $\begin{array}{r}972 \\
28 \\
0\end{array}$ \\
\hline & Total & 991 & 9 & 0 & 1000 \\
\hline \multirow[t]{2}{*}{$\begin{array}{l}\text { (Slides } 1 \text { and } \\
2 \text { combined) }\end{array}$} & $\begin{array}{l}\text { Series } 3 \\
\text { Small } \\
\text { Medium } \\
\text { Large }\end{array}$ & $\begin{array}{c}(4470) \\
54 \\
4\end{array}$ & $\begin{array}{c}(0) \\
21 \\
1\end{array}$ & $\begin{array}{l}(0) \\
3 \\
1\end{array}$ & $\begin{array}{r}4470 \\
78 \\
6\end{array}$ \\
\hline & Total & 4528 & 22 & 4 & 4554 \\
\hline \multicolumn{2}{|c|}{$\begin{array}{l}\text { Gombined total } \\
\text { (Series 1+Series 3) }\end{array}$} & 5519 & 31 & 4 & 5554 \\
\hline \multicolumn{2}{|l|}{ Combined $\%$} & $99 \cdot 37$ & 0.56 & 0.07 & $100 \cdot 00$ \\
\hline \multicolumn{2}{|c|}{$\begin{array}{l}\text { Fiducial limits }(95 \%) \text { of } \% \\
\text { Lower } \\
\text { Upper }\end{array}$} & $\begin{array}{l}99 \cdot 13 \\
99 \cdot 56\end{array}$ & $\begin{array}{l}0 \cdot 38 \\
0 \cdot 79\end{array}$ & $\begin{array}{l}0.02 \\
0.18\end{array}$ & $=$ \\
\hline
\end{tabular}

Each series was based on a single ejaculate from a different individual. The determinations of ploidy are based on FeulgenDNA absorption, except for the numbers in parentheses which were derived from the assumption that all Small nuclei were IN.

Sources of variation in the absorption measurements of IN nuclei were analysed as in the rabbit experiment, the percentage components of variance being: variation including real differences in DNA content of $1 \mathrm{~N}$ nuclei but excluding the two following categories, $89 \%$, differences between batches of size fifty, $6 \%$, lack of repeatability of measurements on the same nuclei, $5 \%$. The $6 \%$ component has a barely significant deviation from zero $(P \sim$ $0 \cdot 05)$. These results are considered in the Discussion.

\section{Human spermatozoa, Series 2}

This series was designed to provide data for estimation of the optimum hydrolysis time in $5 \mathrm{~N}-\mathrm{HCl}$, yielding maximum absorbancy.

Spermatozoa were not visible on the three control slides with zero hydrolysis time. On each of the remaining twenty-seven slides, fifteen nuclei were measured. Of the 405 measurements, six were rejected (three from one slide, one each from three other slides) as being clearly outside the range of other measurements 
on the same slide. Mean values of the remaining measurements on each slide are plotted against log [hydrolysis time] in Text-fig. 3 .

Linear, quadratic, cubic and quartic orthogonal polynomial functions of $\log$ [hydrolysis time] were fitted to the data by least squares regression, and their significance judged from an analysis of variance that led to the following conclusions: (1) there were small but statistically significant differences between slides having the same hydrolysis time; (2) the residual mean square after regression was not significantly greater than the 'between slides within times' mean square, and they were combined to give a pooled 'between slides within times' mean square; (3) the orthogonal polynomial mean squares were tested in turn against this pooled mean square, and all except the quartic term were found to be significant; (4) accepting the reality of the linear, quadratic and cubic terms, the optimum hydrolysis time was estimated as 40.7 min with a $95 \%$ confidence interval from $35.8 \mathrm{~min}$ to $46.1 \mathrm{~min}$. The fitted curve is shown in Text-fig. 3.

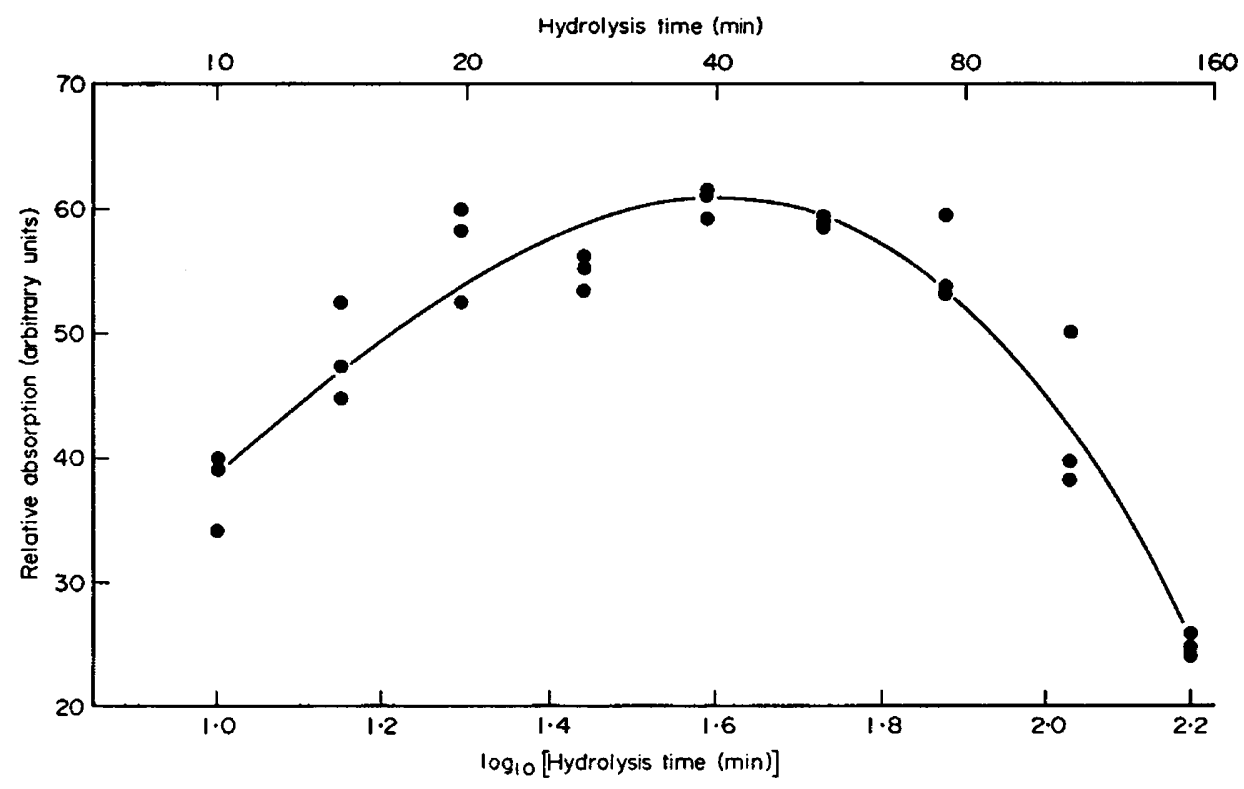

TExT-pig. 3. Series 2. Variation of relative Feulgen-DNA absorption of human sperm nuclei with hydrolysis time in $5 \mathrm{~N}-\mathrm{HCl}$ at $25^{\circ} \mathrm{C}$; single ejaculate with three replicate slides per hydrolysis time; from twelve to fifteen spermatozoa/slide. Each point represents the mean of a single slide and the smooth curve is a third-order polynomial regression on $\log _{10}$ [hydrolysis time].

\section{Human spermatozoa, Series 3}

Two slides that appeared by inspection to have the greatest number of 'acceptable' nuclei were selected from the three replicate slides of Series 2 whose hydrolysis time ( $38.7 \mathrm{~min}$ ) was close to the estimated optimum ( $40.7 \mathrm{~min})$. Nuclei were selected, visually classified and measured for DNA as in previous series, with the single exception that Small nuclei were not measured but simply 
counted. This device, together with the assumption (from the results of Series 1) that all Small nuclei were $1 \mathrm{~N}$, made it possible to determine the ploidy of approximately five times as many spermatozoa in a given time as in previous series. In addition, a number of 'nearest neighbours' (four on the first slide, two on the second) of each Medium and Large nucleus were measured for DNA. These were required to be 'acceptable' but were not otherwise selected and were therefore considered to form a random sample, controlled for possible variations in the depth of staining over the slide, which provided a standard of reference with which to compare the measurements of Medium and Large nuclei.

Slide 1. Out of 3054 nuclei included in the count, fifty-two were Medium and two Large and their absorption measurements (Text-fig. 4) fell into three

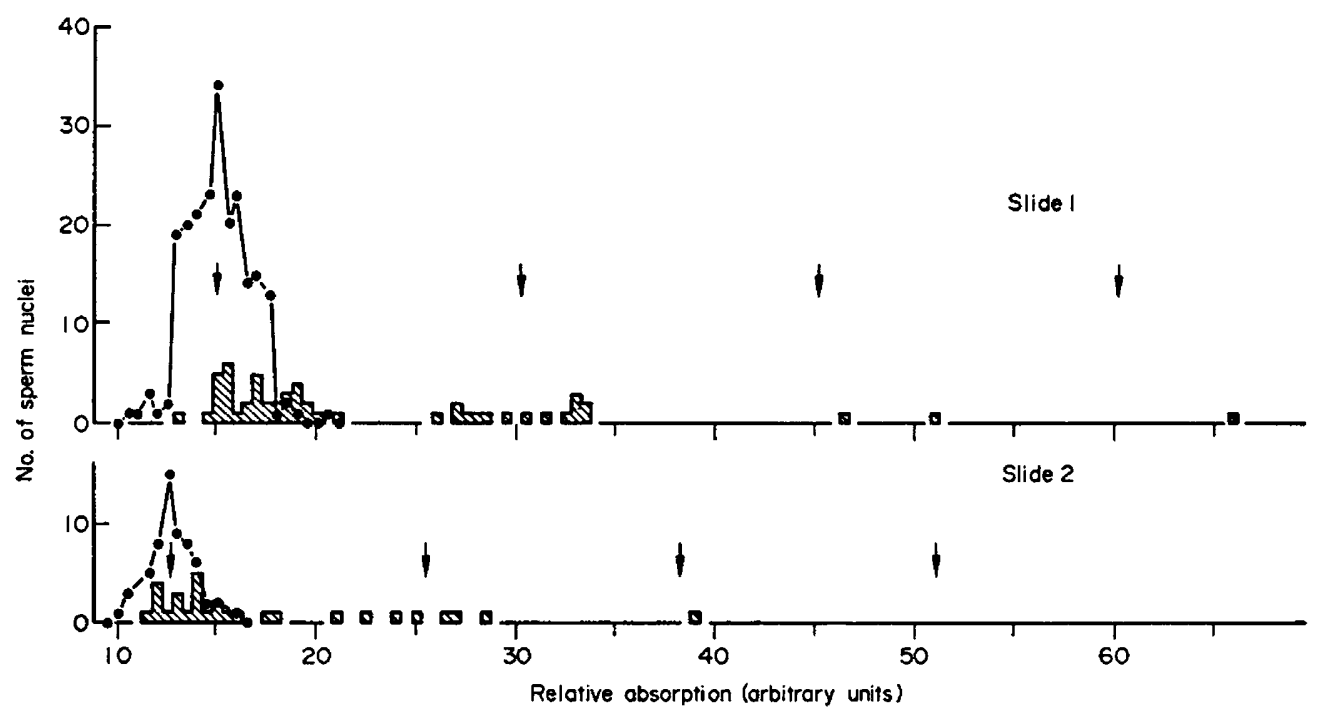

TEXT-FIG. 4. Series 3. Distributions of relative Feulgen-DNA absorption of Medium and Large human sperm nuclei (histograms) and of their 'nearest neighbours' (frequency polygons) on two slides from Series 2 with near-optimal hydrolysis time. Arrows indicate mean relative absorptions of $1 \mathrm{~N}, 2 \mathrm{~N}, 3 \mathrm{~N}$ and $4 \mathrm{~N}$ nuclei, estimated respectively as $1 \times, 2 \times, 3 \times$ and $4 \times$ the mean of the 'nearest neighbours' (which are effectively a random sample of $1 \mathrm{~N}$ nuclei).

groups containing:- (A) thirty-six between 13.0 and 21.0 inclusive, with mean 17.00; (B) fifteen between 26.0 and 33.5 inclusive, with mean 30.27 ; (C) three greater than $46 \cdot 0$, with mean $54 \cdot 5$.

The measurements of the 216 'nearest neighbours' (Text-fig. 4) gave an estimated mean of $15 \cdot 07 \pm 0 \cdot 11$ ( 215 d.f.).

The (A) measurements fell within the range of the 'nearest neighbours' and were therefore assumed to be $1 \mathrm{~N}$. Their mean was significantly higher than that of the 'nearest neighbours', presumably as a result of their having been visually selected for greater size. Noting that the mean of B was almost exactly twice the mean of the 'nearest neighbours', and assuming that the 'nearest neighbours' were a random sample of 'acceptable' spermatozoa and that all polyploid nuclei were Medium or Large, it was concluded that B contained only 
$2 \mathrm{~N}$ nuclei. The $\mathrm{C}$ measurements appeared to be above the $2 \mathrm{~N}$ range but within the expected $3 \mathrm{~N}$ to $4 \mathrm{~N}$ range. Out of a total of 3054 spermatozoa, 3036 were $1 \mathrm{~N}$, fifteen were $2 \mathrm{~N}$ and three were $>2 \mathrm{~N}$. Of the fifty-two Medium nuclei, thirty-five were $1 \mathrm{~N}$, fifteen $2 \mathrm{~N}$ and two $>2 \mathrm{~N}$. Of the two Large nuclei, one was $1 \mathrm{~N}$, none were $2 \mathrm{~N}$ and one was $>2 \mathrm{~N}$.

Slide 2. Out of 1500 nuclei, twenty-six were Medium and four Large and their absorption measurements are given in Text-fig. 4. By reasoning similar to that used for the first slide, it was concluded that 1492 were $1 \mathrm{~N}$, seven were $2 \mathrm{~N}$ and one was $>2 \mathrm{~N}$. Of the twenty-six Medium nuclei, nineteen were $1 \mathrm{~N}$, six were $2 \mathrm{~N}$ and one was $>2 \mathrm{~N}$. Of the four Large nuclei, three were $1 \mathrm{~N}$, one was $2 \mathrm{~N}$ and none were $>2 \mathrm{~N}$. (The lower mean absorption on this slide as compared with the first is probably attributable to fading.)

\section{Human spermatozoa, combined estimates from Series 1 and 3}

The proportions of spermatozoa in all categories were consistent between the two slides of Series 3 and the results of the slides were therefore combined. The combined proportion of $1 \mathrm{~N}: 2 \mathrm{~N}$ was consistent with that obtained from Series 1 (in spite of the sub-optimal hydrolysis time in Series 1 ), but there was a barely significant difference $(P \sim 0.05)$ between the two series in the proportion of Small: Medium. The data from the two series are summarized in Table 1.

\section{Mouse}

Observations were made on 1000 sperm nuclei, using a different and less precise model of the instrument used for the rabbit and human. The absorption of each nucleus was determined ('first measurements'), with a duplicate measurement on every tenth nucleus ('second measurements'). In the rabbit and human series, nuclei had been sampled along a transect taken across the slide, but in the present series batches of fifty consecutive nuclei were measured within small pre-determined rectangular areas. In this way, a correction could be made for variation in the depth of staining over the slide, all fifty measurements in a given batch being multiplied by the same factor to give a batch mean of 100 units. The adjusted data range from 77 to 119 with four 'outliers' at $125,127,134$ and 136. It is, therefore, clear that no nuclei had absorptions in the expected $2 \mathrm{~N}$ or $>2 \mathrm{~N}$ range. It was concluded that all 1000 spermatozoa were $1 \mathrm{~N}$, the upper $95 \%$ fiducial limit for the percentage of $2 \mathrm{~N}$ and $>2 \mathrm{~N}$ being $0.30 \%$. There were no Medium or Large nuclei, though a single Medium nucleus identified outside the random count had an absorption of 221 on the adjusted scale. This was within the expected $2 \mathrm{~N}$ range and indicated that $2 \mathrm{~N}$ mouse spermatozoa are detectable by the method.

The adjusted data were not well fitted by a Normal curve. Even with the four 'outliers' omitted, a $\chi^{2}$ goodness-of-fit test gave a highly significant discrepancy $(P=0.001$, d.f. 31$)$. An analysis of variance of the unadjusted first measurements showed that about $63 \%$ of the total variance was accounted for by differences between batches. With the 'second measurements' included in the analysis, the remaining $37 \%$ could be resolved into a component representing lack of repeatability of measurements on the same nuclei $(7 \%)$ and a component including real variation in DNA content of nuclei $(30 \%)$. 


\section{DISCUSSION}

The reality of polyploid spermatozoa

In previous work on the rabbit and bull, reviewed by Beatty \& Fechheimer (1972), several lines of evidence made it very probable that a proportion of mammalian spermatozoa are diploid. Some of these spermatozoa, even those whose DNA content had been measured, might conceivably have been an arbitrary selection from the tail-end of a unimodal frequency distribution of haploid spermatozoa. This doubt is now dispelled for the rabbit. The frequency distribution of Feulgen-DNA absorption fell into two completely separate groups, a major one (haploids), and a smaller one (clearly of diploids) with a mean DNA absorption nearly twice that of the major group. In human material, the groups were virtually discrete; again, one small group (diploids) had almost exactly twice the mean DNA absorption of the major group, and there was a third very small group (presumed to be triploids and/or tetraploids) with DNA values about three to four times that of the major group. In the mouse, only a single spermatozoon was interpreted as diploid, but Stolla \& Gropp (1974), in selected 'malformed' spermatozoa of an $F_{1}$ cross of $M u s$ musculus $\times M$. poschiavinus, demonstrated a small and nearly discrete group whose mean DNA absorption was almost exactly twice the normal level.

\section{The determination of ploidy in individual spermatozoa}

Our primary means of identification was based on DNA absorption, but spermatozoa were also classed subjectively as Small, Medium or Large according to the size of the head in nigrosin-eosin preparations or the size of the nucleus in Feulgen preparations. The present work shows that the Small and Medium classes in the rabbit corresponded exactly with haploids and diploids. In this species, therefore, we can say (within the limits of our sampling error) individual haploid and diploid spermatozoa can be identified merely by looking at them. Data on the equivalence of Large spermatozoa with triploids or tetraploids are not yet available in rabbits.

In man, however, the correspondence between visually-appraised classes and DNA-scored ploidy is not perfect. Although all Small nuclei that were measured for DNA had an absorption in the $1 \mathrm{~N}$ range, $69 \%$ of the Medium nuclei were $1 \mathrm{~N}$ and only $28 \%$ were $2 \mathrm{~N}$ (with $3 \%>2 \mathrm{~N}$ ), while of the few Large nuclei, four were $1 \mathrm{~N}$, one was $2 \mathrm{~N}$ and one was $>2 \mathrm{~N}$. We agree with Sumner (1971) that "... almost all the polyploid sperm found by DNA measurement were easily recognisable visually by their larger areas ...". The converse is not true; we found that only a minority of spermatozoa scored as having a larger area in optical projection (Medium or Large) were polyploid. Data involving ' $\mathrm{F}$-bodies' instead of DNA measurement also indicate that many Medium spermatozoa are haploid and not diploid (Beatty, 1974). Visual appraisal of size is, therefore, only partly a guide to the ploidy of human spermatozoa. This would remain so even if the subjective threshold separating Small from Medium nuclei were raised, since some $2 \mathrm{~N}$ nuclei definitely appeared smaller than some $1 \mathrm{~N}$. The best we can do is to form a corrective factor applicable to the particular observer: in 
our observations, about one third of Medium spermatozoa are to be considered $2 \mathrm{~N}$, about $3 \%>2 \mathrm{~N}$, and the remainder $1 \mathrm{~N}$. The scanty data for Large spermatozoa indicate that most are $1 \mathrm{~N}$ while some are $2 \mathrm{~N}$ and $>2 \mathrm{~N}$.

We believe that detailed study of our observed distributions of DNA absorption does not permit interpretation of the distributional 'tails' in terms of aneuploidy, and in addition we cannot support or reject either of the hypotheses that the 'true' haploid distribution is (1) a single Normal distribution, or (2) a compound of equal Normal distributions of X-and Y-bearing spermatozoa differing slightly in their means. This is because the analyses of variance revealed large sources of technical error, manifested by differences between batches (attributable to variation in the depth of staining on a slide, visually obvious in the mouse series), and by lack of repeatability of measurements on the same nuclei (attributable to instrument error).

\section{The incidence of ploidy classes of spermatozoa}

The incidence of $1.09 \% 2 \mathrm{~N}$ spermatozoa in a young (35-week-old) ADstrain male rabbit specially chosen to show a high incidence was of the order expected, but was not a measure of the incidence among rabbits in general, for which age and strain effects gave a variation from about $0.1 \%$ to about $1.5 \%$ (Fig. 2, Beatty \& Fechheimer, 1972). Accepting (as a result of the present work) the equivalence of Small with $1 \mathrm{~N}$ and Medium with $2 \mathrm{~N}$, and assuming an equivalence of Large with $>2 \mathrm{~N}$, then a survey of 138001 spermatozoa of 69 29-week-old AS-strain rabbits (partly described by Beatty \& Fechheimer, 1972) showed an incidence of $99.691 \% 1 \mathrm{~N}$ (137575 Small), $0.308 \% 2 \mathrm{~N}$ (425 Medium) and $0.001 \%>2 \mathrm{~N}$ (one Large).

Data on 1670 spermatozoa from four patients at a sub-fertility clinic (Sumner, 1971) gave the following estimates: $98.98 \%$ haploid, $0.96 \%$ diploid, $0.06 \%$ tetraploid. Our figures from two presumptively normal subjects were of the same order: out of 5554 spermatozoa, $99.37 \% 1 \mathrm{~N}, 0.56 \% 2 \mathrm{~N}, 0.07 \%>2 \mathrm{~N}$.

Diploid spermatozoa appear to be rare in mice. Stolla \& Gropp (1974) report none among 300 spermatozoa from each of the species $\boldsymbol{M}$. musculus and $M$. poschiavinus, and we report none among 1000 randomly sampled $M$. musculus spermatozoa. The rarity of $2 \mathrm{~N}$ mouse spermatozoa in our material is the more striking in that we had deliberately chosen material that might have a high incidence.

\section{The origin of ploidy classes of spermatozoa}

Although it is clear from the results that $2 \mathrm{~N}$ spermatozoa exist, and reasonably clear that $3 \mathrm{~N}$ and/or $4 \mathrm{~N}$ spermatozoa occur, we do not have enough data to estimate the relative proportions of $3 \mathrm{~N}$ and $4 \mathrm{~N}$. Because of this uncertainty, we can draw only conditional conclusions in attempting to distinguish between two possible modes of origin of extra chromosome sets: (a) 'coalescence' of cells that have already divided, and (b) 'chromosome doubling' by a failure of cytoplasmic division so that two sets of chromosomes remain in one cell instead of being partitioned among two. Both modes of origin, however, involve the existence of some tetraploid spermatozoa. 
We may first assume that cells have 'coalesced', giving cells of all ploidy types with relative frequency probably related inversely to the ploidy. Inspection of our Text-fig. 4 does suggest in man that both triploids and tetraploids exist in addition to the obvious diploids, and that frequency is inversely related to ploidy.

Alternatively, we may assume that 'chromosome doubling' occurs at either meiotic division, giving diploid spermatozoa, while successive failure at both meiotic divisions would give tetraploidy. Triploidy should not arise. If failure of the second division occurs independently of the first and with equal frequency (Q), it may be calculated from the 138001 rabbit spermatozoa quoted above that $Q=0.31 \%$ and the 'expected' number of tetraploids $(0.3)$ is of the same order as the observed number (1) of Large spermatozoa. In man, a similar analysis of the 5554 spermatozoa in the present work gave $Q=0.70$, and an 'expected' 0.07 tetraploids. The four spermatozoa observed to have a ploidy greater than two would all have been tetraploids under the assumption of 'chromosome doubling'. Even if we take the estimated value of $Q$ at its upper $99 \%$ confidence limit (to allow for sampling variation), it can be calculated that the probability of obtaining four or more tetraploids is low $(P<0.0001)$. Evidently, in man, more presumed tetraploids were encountered than expected. No explanation can be found by supposing that $Q$ is not the same at the first and second meiotic divisions. The more they differ, the greater will be the lack of fit. Possible explanations are that 'chromosome doubling' in man is not independent at the two divisions, or that some or all of the presumed tetraploids are really triploids arising from 'coalescence'.

\section{The fate of diploid spermatozoa}

The fate of diploid spermatozoa is still somewhat obscure. Experimental alterations in the proportion of diploid rabbit spermatozoa, followed by artificial insemination, did not change the proportion of triploid embryos (Fechheimer \& Beatty, 1974). This indicated that diploid spermatozoa may have a reduced chance of fertilizing an egg and may fail to contribute substantially to the incidence of triploidy.

The indications in man are that some diploid spermatozoa may be fertile. The present work gives an estimated incidence of $0.56 \% 2 \mathrm{~N}$ human spermatozoa which (if normally fertile) might be expected to yield $0.56 \%$ triploid embryos. A preliminary estimate exists that $60 \%$ of human triploidy is attributable to 'chromosome doubling' in the egg and $40 \%$ to fertilization of a normal egg by diploid spermatozoa (Beatty, 1974). By simple proportion, therefore, we might expect $0.56 \%$ triploids arising from fertilization with diploid spermatozoa to be accompanied by $60 / 40 \times 0.56 \%=0.84 \%$ of triploids arising from chromosome doubling in the egg. The sum of $0.56 \%$ and $0.84 \%(=1.40 \%)$ is encouragingly close to the estimated $1.20 \%$ of triploids among recognized conceptions in man (Jacobs, 1972).

The low incidence of diploid spermatozoa in mice suggests that they do not play a major role in the incidence of triploidy. Further, Krzanowska (1974) found that diploid spermatozoa (scored by visual appraisal of size) are not found in the oviduct and may have been eliminated at the uterotubal junction. 


\section{ACKNOWLEDGMENTS}

We are indebted to $\mathrm{Mr} \mathrm{V}$. J. Coulter for skilled technical assistance, and to Dr R. V. Short and Mr D. W. Richardson of the M.R.C. Unit for Reproductive Biology for access to human semen samples. Dr R. F. Lyndon of the Department of Botany (Edinburgh) kindly allowed us to use his densitometer, and we are grateful to Dr G. W. Ashcroft and Mr R. C. Dow of the M.R.C. Brain Metabolism Unit for the loan of another machine. The work was supported by a grant from The Ford Foundation.

\section{REFERENCES}

BeAtty, R. A. (1957) Parthenogenesis and Polyploidy in Mammalian Development. Cambridge University Press. Beatty, R. A. (1974) Genetic aspects of spermatozoa. In Physiology and Genetics of Reproduction, Part A, pp. 183-196. Eds E. M. Goutinho \& F. Fuchs. Plenum Press, New York.

Beatty, R. A. \& FechHeimer, N.S. (1972) Diploid spermatozoa in rabbit semen and their experimental separation from haploid spermatozoa. Biol. Reprod. 7, 267-277.

Beatty, R. A. \& Sharma, K. N. (1960) Genetics of gametes. III. Strain differences in spermatozoa from eight inbred strains of mice. Proc. R. Soc. Edinb. B, 68, 25-53.

Böhm, N., Sprenger, E., Schlüter, G. \& Sandritter, W. (1968) Proportionalitätsfehler bei der Feulgen-Hydrolyse. Histochemie, 15, 194-203.

Boute, A. \& Boué, J. (1974) Chromosome abnormalities and abortion. In Physiology and Genetics of Reproduction, Part B, pp. 317-339. Eds E. M. Coutinho \& F. Fuchs. Plenum Press, New York.

Carr, D. H. (1971) Ghromosome studies in selected spontaneous abortions. Polyploidy in man. 7. med. Genet. 8, 164-174.

Deeley, E. M. (1955) An integrating microdensitometer for biological cells. F. sci. Instr. $32,263$.

Fechheimer, N. S. \& BeATTY, R. A. (1974) Chromosomal abnormalities and sex ratio in rabbit blastocysts. 7. Reprod. Fert. 37, 331-341.

Fisher, R. A. \& YAtes, F. (1963) Statistical Tables. Oliver \& Boyd, Edinburgh and London.

Jacobs, P. A. (1972) Ghromosome abnormalities and fertility in man. In The Genetics of the Spermatozoon, pp. 346-358. Eds. and publishers, R. A. Beatty (Edinburgh) and S. Gluecksohn-Waelsch (New York). Departments of Genetics of The University of Edinburgh and the Albert Einstein College of Medicine.

KRZANowsKa, H. (1974) The passage of abnormal spermatozoa through the uterotubal junction of the mouse. F. Reprod. Fert. 38, 81-90.

Moore, G. P. M. \& BeAtTy, R. A. (1974) The DNA content of spermatozoa from different strains of mice. 7. Reprod. Fert. 38, 473-476.

Stolla, R. \& GROPP, A. (1974) Variation of the DNA content of morphologically normal and abnormal spermatozoa in mice susceptible to irregular meiotic segregation. $\mathcal{F}$. Reprod. Fert. 38, $335-346$.

SumNer, A. T. (1971) Frequency of polyploid spermatozoa in man. Nature, Lond. 231, 49. 